\title{
Giant sulfur bacteria host endobiont bacteria in intracellular vesicles
}

\author{
BEVERLY FLOOD, DALTON LEPRICH AND JAKE \\ BAILEY
}

University of Minnesota

Presenting Author: baileyj@umn.edu

Eukaryotic organelles such as mitochondria and chloroplasts are thought to be derived from symbiotic bacteria that were hosted by a proto-eukaryotic microbe in the deep evolutionary past. One challenge associated with this theory is arriving at a mechanism by which one prokaryote was internalized by another prokaryote. Prokaryotes are not commonly known to have the capacity for bringing material into the cell via endocytosis. Indeed, despite their potential relevance to eukaryote evolution, endosymbiotic relationships involving prokaryotic host cells in the modern biosphere are extremely rare, and not well understood. Parasites of mealybug symbionts are the only clear example of a bacterial-bacterial endosymbiosis that is well studied.

We discovered a population of giant sulfur bacteria cells, Thiomargarita spp., that harbor dense populations of intracellular bacteria. Molecular evidence shows that at least some of these endobiont bacteria are putative sulfur-oxidizing bacteria that are closely related to gammaproteobacterial endosymbionts of siboglinid worms. We observed that the endobiont cells commonly occur within novel acidic vesicle-like features within the Thiomargarita host cell. While the ancestral eukaryotic host cell was unlikely to have been a giant gammaproteobacterium, investigating the nature of this endobiont/host relationship, as well as how these vesicles form and function, may tell us more about potential mechanisms of membrane remodeling, compartmentalization, and endobiont internalization in prokaryote host cells - all fundamental processes that are thought to have been critical to the evolution of eukaryotes. 